\section{Diagnostic accuracy of keratoconus using anterior segment optical coherence tomography}

\author{
Pinakin G. Davey, ${ }^{1,2}$ Christina Newman, ${ }^{2}$ \\ Anna Ablamowicz, ${ }^{2}$ Daniel Fuller ${ }^{2}$ \\ ${ }^{1}$ College of Optometry, Western \\ University of Health Sciences, Pomona, \\ CA; ${ }^{2}$ Department of Research, Southern \\ College of Optometry, Memphis, TN, USA
}

\begin{abstract}
The aims of the present study are i) to evaluate the diagnostic accuracy of RTVue noncontact, high-resolution spectral domain optical coherence tomography (RTVue OCT) in identifying keratoconic eyes from a group of healthy eyes; and ii) to examine how the severity of disease affects diagnostic accuracy. Corneal thickness measurements were performed using the RTVue OCT on 58 eyes of 29 individuals (10 males, 19 females). Of them, 28 eyes were deemed to be keratoconic, and 30 eyes healthy on the basis of clinical findings. Sensitivity, specificity and receiver operating characteristic (ROC) area was calculated overall and by grouping eyes by severity of disease. On the whole, the ROC area was highest for the parameters minimum thickness and difference between minimum and maximum thickness of cornea (0.98). The ROC area for the parameters difference in superior nasal and inferior temporal thickness and difference in superior and inferior thickness was 0.81 and 0.77 , respectively. The ROC area in identifying eyes with keratoconus varied as a function of severity of disease. From our analysis we can conclude that the RTVue OCT provides excellent diagnostic ability in identifying moderate to advanced cases of keratoconus.
\end{abstract}

\section{Introduction}

Refractive surgery, the management of corneal diseases like keratoconus and contact lens problems, the postoperative management of penetrating keratoplasty, and now also the management of patients with glaucoma and ocular hypertension have made accurate measurements of corneal thickness of prime concern. ${ }^{1-4}$ The process of measuring central corneal thickness (pachymetry) can be carried out optically - including specular microscopy, ${ }^{5}$ optical coherence tomography, ${ }^{6}$ and Scheimpflug imaging - or by using ultrasound. Our prior work has shown that although the ultrasound pachymeter is highly repeatable and reproducible, ${ }^{7}$ the technique and number of attempts involved in measuring central corneal thickness could influence the outcome of the results. ${ }^{8}$ Recently, RTVue cornea module (Optovue Inc., Fremont, CA, USA), i.e. a noncontact, high-resolution spectral domain optical coherence tomography (RTVue OCT) that measures corneal thickness, has become available. Previous work from Gunvant and Darner ${ }^{9}$ has showed that there are significant differences in measurement values both in central and peripheral cornea when using ultrasonic pachymeter and RTVue OCT.

Keratoconus is a common disorder that is especially prevalent in western communities (approximately $50-200 / 100,000$ people). ${ }^{10}$ Keratoconus is characterized by corneal thinning and irregular astigmatism. The optical coherence tomography gives an advantage of measuring a central $6 \mathrm{~mm} \times 6 \mathrm{~mm}$ region of cornea (Figure 1) compared to ultrasonic pachymeter that only gives a point measurement. Furthermore, the optical coherence tomography gives a non-contact measurement, whereas the ultrasonic measurements requires contact with cornea. The RTVue provides a suggested criterion to keratoconus screening (Table 1). Using this suggested criterion, Gunvant and Darner ${ }^{9}$ showed that false positive rate (specificity) was higher when using classifiers individually. Moreover, combining the criterion gave even better specificity. The aim of this study was twofold: i) evaluate the diagnostic accuracy of RTVue OCT in identifying keratoconic eyes from a group of healthy eyes; and ii) evaluate the diagnostic accuracy of RTVue OCT as a function of severity of the disease.

\section{Materials and Methods}

\section{Subjects}

Fifty eight eyes of 29 subjects (10 males, 19 females), whose mean age was 31.43 years (range 20-61.9), were examined for the study. Twenty-eight eyes were deemed to be keratoconic and 30 eyes healthy on the basis of clinical criteria of a collaborative longitudinal evaluation of keratoconus (CLEK) study ${ }^{11}$ by two experienced clinicians (CN and DF). Classification and severity were graded by obtaining the simulated keratometry (Sim K) findings from the $3.0 \mathrm{~mm}$ annulus on the Medmont topographer.

A total of 29 subjects were evaluated during the course of study. A control group of 15 normal subjects was examined. These patients were chosen based on their lack of previous diagnosis of corneal disease. A total number of 14 patients (28 eyes) with diagnosis of keratoconus were included in the study as they had
Correspondence: Pinakin G. Davey, College of Optometry, Western University of Health Sciences, 309 E. Second Street, 91766-1854 Pomona, CA, USA.

Tel. +1.909.623.6116 - Fax: +1.909.469.5535.

E-mail: contact@pinakin-gunvant.com

Key words: keratoconus, corneal thickness, optical coherence tomography, diagnostic accuracy, screening.

Contributions: the authors contributed equally.

Conflict of interests: the authors declare no potential conflict of interests.

Received for publication: 10 August 2012.

Revision received: 22 January 2013.

Accepted for publication: 20 March 2013.

This work is licensed under a Creative Commons Attribution NonCommercial 3.0 License (CC BYNC 3.0).

(C) Copyright P.G. Davey et al., 2013

Licensee PAGEPress, Italy

Optometry Reports 2013; 3:e2

doi:10.4081/optometry.2013.e2

no prior history of ocular surgery. All ethnicities and genders were included from the eye center (TEC) population.

Fourteen patients with a diagnosis of keratoconus and 15 normal subjects were included in the study. In the keratoconus group (11 females, 3 males), all but one of the subjects had keratoconus in both eyes. Eight of those had an advanced presentation of the disease in both eyes and two were classified with moderate severity in both eyes. Since keratoconus is an asymmetrical bilateral disease, four of the subjects had a different stage of severity between their eyes. Two subjects had moderate severity OD and advanced presentation OS, 1 subject had an early form of keratoconus OD and moderate severity $\mathrm{OS}$. The remaining subject had an early presentation in his OD only and no classification OS. All 30 eyes of the control group (8 females, 7 males) were included and considered to be healthy.

The study was approved by the institutional review board of both the College of Optometry (Western University of Health Sciences, Pomona, CA, USA) and the Department of Research (Southern College of Optometry, Memphis, TN, USA). All participants provided informed consent and the study protocols adhered to the provisions of the Declaration of Helsinki.

Measurement performed using the cornea module of RTVue optical coherence tomography

The RTVue OCT is designed for imaging the 
posterior parts of the eye (retina and optic disc) and is modified using a wide-angle lens measuring scan wide up to $6 \mathrm{~mm}$ and having a transverse resolution of 15 microns. The raw images obtained by the cornea module are warped due to refraction at the cornea air interface. The images are un-warped automatically by software to obtain thickness measurements. The pachymetry protocol was utilized to obtain the corneal thickness in the central and the mid-peripheral regions. The pachymetry map consists of eight meridional scans that take about $0.31 \mathrm{sec}$ to be acquired (Figure 2). The pupil was used as an anatomical landmark to align the scan centrally. The 6-mm pachymetry map is obtained by interpolation of the thickness profiles of the eight meridians (Figure 2). The Optovue cornea module could be used for screening keratoconus and the recommended guidelines are given in Table 1 (Optovue Inc.). These values were utilized for study purposes and measurements were performed by an experienced observer (CN).

\section{Statistical analysis}

The ROC area was calculated using Analyzeit for Excel V2.22 for each parameter in identifying eyes with early, moderate and severe keratoconus.

\section{Results}

Table 2 gives the overall ROC area and vari- ous severity of the disease. As can be seen, the ROC area is lower in early disease and higher in advanced cases of disease. This is not surprising given that in advanced cases of disease it is easier to diagnose the problem. The parameter difference in superior and inferior thickness does not follow the above mentioned pattern because in advanced cases of disease the cornea is thinner throughout and the difference in superior $v s$ inferior thickness becomes lesser and lesser. In such conditions, minimum thickness of cornea may be a better indicator.

\section{Discussion}

Keratoconus is characterized by corneal thinning and irregular astigmatism. ${ }^{9}$ Ideally, keratoconus screening should include both corneal topography and corneal thickness measurements. In this study we found that in eyes with moderate to advanced keratoconus, RTVue OCT parameters were on average 80 $90 \%$ accurate in diagnosis without using any information of corneal topography. Hence, in moderate to advanced cases of keratoconus information of corneal topography may not add benefit diagnostically.

The diagnostic ability in early stages of disease was lower about $70 \%$ on average. This result should be interpreted with caution as the number of cases with early keratoconus was less than moderate and severe groups. The group with early keratoconus is also most likely to benefit from a combination of criteria (Table 1).

\section{Conclusions}

To conclude, our considerations make it clear that further studies combining both corneal topography and corneal thickness measurements are thus required to produce a global classifier.

\section{References}

1. Thornton SP. A guide to pachymeters. Ophthalmic Surg Las 1984;15:993-5.

2. Gordon M0, Beiser JA, Brandt JD, et al. The ocular hypertension treatment study: baseline factors that predict the onset of primary open-angle glaucoma. Arch Ophthalmol-Chic 2002;120:714-20.

Figure 2. Pachymetry map of corneal thickness showing the central and peripheral measurements obtained by the cornea module of RTVue optical coherence tomography.
3. Kass MA, Heuer DK, Higginbotham EJ, et al. The ocular hypertension treatment study: a randomized trial determines that
Figure 1. Optical coherence tomography of measurement centered on the cornea, using pupil as an anatomical landmark. 
topical ocular hypotensive medication delays or prevents the onset of primary open-angle glaucoma. Arch OphthalmolChic 2002;120:701-13.

4. Palmberg P. Answers from the ocular hypertension treatment study. Arch Ophthalmol-Chic 2002;120:829-30.

5. Wheeler NC, Morantes CM, Kristensen RM, et al. Reliability coefficients of three corneal pachymeters. Am J Ophthalmol 1992;113:645-51.

6. Muscat S, McKay N, Parks S, et al. Repeatability and reproducibility of corneal thickness measurements by optical coherence tomography. Invest Ophth
Vis Sci 2002;43:1791-5.

7. Gunvant P, Broadway DC, Watkins RJ. Repeatability and reproducibility of the BVI ultrasonic pachymeter. Eye 2003; 17:825-8.

8. Haun A, Gunvant P, Baskaran M, Vijaya L. Central corneal thickness measurement using a pachometer: Mean or lowest values? Invest Ophth Vis Sci 2004;45: 137(Abstract).

9. Gunvant P, Darner R. Evaluation of corneal thickness measurements obtained using optical coherence tomography and ultrasound technique and determination of specificity in keratoconus screening. In:
Manning DJ and Abbey CK, eds. Medical imaging 2011: image perception, observer performance and technology assessment. Proceedings of the SPIE Conference Volume 7966, 2011 Feb 12-17, Lake Buena Vista (Orlando), FL, USA. Bellingham, WA: SPIE ed.; 2011. pp 1-8.

10. Coster D. Cornea fundamentals of clinical ophthalmology series. London: BMJ Books Publ.; 2001.

11. Zadnik K, Barr, JT, Edrington TB, et al. Baseline findings in the collaborative longitudinal evaluation of keratoconus (CLEK) study. Invest Ophth Vis Sci 1998; 39:2537-46. 